\title{
How to compute the thermodynamics of a glass using a cloned liquid
}

\author{
Marc Mézard ${ }^{\mathrm{a}, \mathrm{b}, *}$ \\ anstitute for Theoretical Physics, University of California, Santa Barbara, CA 93106-4030, USA \\ ${ }^{\mathrm{b}}$ Physique Théorique-ENS, CNRS, France
}

Received 15 December 1998

\begin{abstract}
The recently proposed strategy for studying the equilibrium thermodynamics of the glass phase using a molecular liquid is reviewed and tested in details on the solvable case of the $p$-spin model. We derive the general phase diagram, and confirm the validity of this procedure. We point out the efficacy of a system of two weakly coupled copies in order to identify the glass transition, and the necessity to study a system with $m<1$ copies ('clones') of the original problem in order to derive the thermodynamic properties of the glass phase. (c) 1999 Published by Elsevier Science B.V. All rights reserved.
\end{abstract}

Keywords: Glass phase; Cloned liquid; $p$-spin glass; Phase diagram

\section{Introduction}

It has been proposed recently that the thermodynamic properties of the glass phase can be deduced from those of the corresponding liquid phase, computed above the glass transition but for a 'replicated' liquid involving $m$ species with $m<1$, forming then a molecular bound state [1]. This scheme is appealing in that it provides a general strategy for computing the equilibrium properties of the glass phase. ${ }^{1}$ This whole strategy is supposed to be valid whenever the thermodynamic transition is due to an 'entropy crisis' scenario [2-10]. ${ }^{2}$ This basically means that the configurational

\footnotetext{
* Correspondence address: Institute for Theoretical Physics, University of California, Santa Barbara, CA 93106-4030, USA. Fax: +1-805-893-2431; e-mail: mezard@itp.ucsb.edu.

${ }^{1}$ Of course experimental glasses are in general not in equilibrium, but the equilibrium can be reached in some cases with carefully conducted simulations, and it is also useful to first study in details the equilibrium properties before turning to a dynamical study.

${ }^{2}$ Recent reviews can be found in Refs. [11,12]. An introduction to the theory is given in Ref. [13]. Some introduction to the very recent developments in connection with the spin glass ideas is given in Ref. [14].
} 
entropy defined as the logarithm of the number of accessible metastable states of the system, which is extensive above the transition temperature, becomes zero below the transition temperature.

The archetypes of such systems, and the only ones for which one can get a full control so far, are provided by the so-called $p$-spin models, some infinite range spin glasses involving $p>2$ spin interactions, which have been already much studied. In this paper we want to test the general strategy of [1] on these exactly solvable cases. We show explicitely how the properties of the glass phase in these systems can be deduced from that of the replicated liquid, and we point out some subtleties of the phase diagram of this replicated liquid, which should be kept in mind when one deals with the real systems of interacting particles.

This paper complements the work by Franz and Parisi [36] which performed a similar study of the phase diagram of two coupled systems when one of the system is thermalized in the low-temperature phase. Here we concentrate instead on the case where we use an arbitrary number $m$ of coupled systems which are all identical, as used in [1] (The case $m=2$ was alluded to in [36]).

\section{The basic strategy}

\subsection{Identifying the glass phase}

Let us assume that there exists a glass phase in some system. How can one distinguish the glass phase from the liquid one, in the framework of equilibrium statistical mechanics? A simple answer, developed initially in the spin glass context [16-19], is the following. Take two copies, with a small attraction between particles in the two copies, of strength $\varepsilon$. The attraction is short range (range less or equal to the typical interparticle distance), and it couples all particles of system 1 to all particles of system 2 . Look at the correlations between the two copies when $\varepsilon \rightarrow 0$ (limit taken after the thermodynamic limit). If there remain some correlations in this double limit, this means that the system is in a solid phase (a glass or a crystal); otherwise it is in the liquid phase. This general technique is a very robust one which is able to identify the glass phase in very diverse systems, ranging from spin glasses to directed polymers and to structural glasses $[16-18,20,1]$.

In structural glasses this transition is associated with a spontaneous breaking of a translation symmetry. For $\varepsilon=0$, the Hamiltonian is invariant by the relative global translation of the particles in the second system relative to the particles in the first one (assuming periodic boundary conditions). This symmetry is spontaneously broken in the solid phase. Therefore, one expects that there is no way in which one can connect continuously the liquid phase to the glass phase without encountering a phase transition. We shall check this property explicitely on the case of the $p$-spin mean-field spin glass: we shall work out the phase diagram in the plane $\varepsilon, T$, showing the existence of a glass transition temperature at all $\varepsilon$. 


\subsection{Nature of the glass transition in the discontinuous case}

This identification of the transition from the study of two coupled systems does not allow to reach the properties of the low-temperature phase from a study of the high-temperature one, precisely because of the existence of a phase transition. It turns out that, for a specific class of systems, there exists a more subtle generalization of this approach which uses an arbitrary number $m$ of coupled systems and allows to study the glass phase. These are the systems for which the glass transition is of a discontinuous type, called 'one-step replica symmetry breaking' (one step rsb) in the spin glass language. Basically, this situation is encountered whenever there exist some low-lying states of the system which are well separated in phase space and have independent free energies [21], a situation which has been argued to give a good description of the structural glass phase [15]. More precisely, the extensive part of their free energies are equal, but the fluctuating, non-extensive part of their free energies are not correlated from state to state. One may expect that such systems will be generic in the sense that this situation corresponds to a universality class for extreme event statistics [22]. Proving whether a given physical system actually falls in such a class may be quite difficult, but a useful approach, adopted in [1], consists in assuming that it does, computing the properties of the system within such an assumption, and comparing them to experimental and numerical data. The success of this strategy, and the number of experimental obsevations that it explains and relates, have added confidence to the validity of the old conjecture [7-10] according to which fragile structural glasses fall into this category $[15,1]$.

In this paper we shall concentrate on the mean-field spin glasses with $p$-spin interactions $(p>2)$ which have been shown to belong to this class of systems, both for Ising spins and for spherical spins [6-10,23,24]. (Several other sytems have been proven to belong to this class, including some spin glasses without quenched disorder [25-29].) We shall briefly review some of their properties which are crucial for understanding the nature of their glass transition and how the glass phase can be studied. These systems possess an infinity of metastable states, which we shall label by an index $\alpha$. Each state is characterized [30] by the value of the local magnetization on each site, $m_{i}^{\alpha}=\left\langle s_{i}\right\rangle_{\alpha}$. The states, and their free energies, can be defined as solutions of TAP equations. A very important quantity is the number $\mathscr{N}$ of such metastable states with a given free energy density $f$. It turns out that, in some range of free energies, this number is exponentially large in the number of degrees of freedom. One can thus define a corresponding entropy, called the configurational entropy in the glass litterature and the complexity in the spin glass litterature [31-33,6], defined by

$$
\mathscr{N}(f, T, N) \approx \exp \left(N S_{c}(f, T)\right),
$$

The number of metastable states vanishes outside of the region $f_{\min }(T)<f<f_{\max }(T)$, and the configurational entropy $S_{c}(f, T)$ goes to zero at $f_{\min }(T)$.

Let us discuss the properties of such a system at thermal equilibrium. We call $f_{\alpha}$ the free-energy density of state $\alpha$. At low enough temperatures the total free-energy 
density of the system $\left(f_{S}\right)$ can be well approximated by the sum of the contributions to the free energy of each particular minimum

$$
Z \equiv \exp \left(-\beta N f_{S}\right) \simeq \sum_{\alpha} \exp \left(-\beta N f_{\alpha}\right)
$$

For large values of $N$ we can write

$$
\exp \left(-N \beta f_{S}\right) \approx \int_{f_{\min }}^{f_{\max }} \mathrm{d} f \exp \left(-N\left(\beta f-S_{c}(f, T)\right) .\right.
$$

Using the saddle-point method we find that

$$
f_{S}=\min _{f} \Phi(f) \equiv f^{*}-T S_{c}\left(f^{*}, T\right),
$$

where

$$
\Phi(f) \equiv f-T S_{c}(f, T) .
$$

This formula is quite similar to the usual formula for the free energy, i.e. $f=\min _{E}(E-$ $T S(E)$ ), where $S(E)$ is the entropy density as a function of the energy density. The main difference is the fact that the total entropy of the system has been decomposed into the contribution due to small fluctuations around a given configuration (this piece has been included into $f$ ), and the contribution due to the existence of a large number of locally stable configurations, the configurational entropy.

Calling $f^{*}$ the value of $f$ which minimizes $\Phi(f)$, we have two possibilities:

- The minimum of $\Phi$ lies inside the interval and it can be found as the solution of the equation $\beta=\partial S_{c} / \partial f$. In this case we have

$$
\Phi=f^{*}-T S_{c}^{*}, \quad S_{c}^{*}=S_{c}\left(f^{*}, T\right) .
$$

The system may stay in one of the many possible minima. The number of accessible minima is $\exp \left(N S_{c}^{*}\right)$. The entropy of the system is thus the sum of the entropy of a typical minimum and of $S_{c}^{*}$, which is the contribution to the entropy coming from the exponentially large number of metastable configurations.

- The minimum of $\Phi$ is at the extreme value of the range of variability of $f$ : it sticks at $f^{*}=f_{\min }$ and the total free-energy is $\Phi=f_{\min }$. In this case the contribution of the configurational entropy to the free energy is zero. The different states which contribute to the free energy have a difference in free-energy density which is of order $N^{-1}$ (a difference in total free energy of order 1), and these free energy differences are uncorrelated and exponentially distributed [21,30], corresponding to Gumbel's distribution of extremes [22].

Therefore, the glass transition of this system is a thermodynamic transition associated to what is sometimes called an 'entropy crisis': the entropy associated with the number of metastable states which contribute to the partition function vanishes below the transition temperature. The transition is of second order from the thermodynamic point of view, having a continuous entropy and a jump in specific heat, but it is associated with a discontinuity of the order parameter [6]. We call such a transition a 'discontinuous glass transition'. 


\subsection{Cloning}

Clearly, the equilibrium thermodynamics of this class of glasses is fully described by the knowledge of the configurational entropy as a function of temperature and free-energy density. Monasson [34] proposed the following method to compute this function. Let us consider $m$ copies of the system which are constrained to stay in the same minimum. We shall discuss below how one can achieve this constraint, but let us first discuss the physics of this constrained system. Its partition function is basically the Laplace transform of the number of states

$$
Z_{m}=\int_{f_{m}}^{f_{M}} \mathrm{~d} f \mathrm{e}^{-N\left[m \beta f-S_{c}(f, T)\right]} .
$$

The free energy per spin,

$$
\phi(m, T)=-\frac{1}{\beta m N} \log Z_{m} \approx \min _{f}\left(f-\frac{T}{m} S_{c}(f, T)\right),
$$

allows to compute the configurational entropy $S_{c}(f, T)$. Because of the concavity of the $S_{c}$ versus $f$ curve, its largest possible slope is

$$
\alpha(T) \equiv \frac{\partial S_{c}}{\partial f}\left(f_{\min }, T\right) .
$$

Performing the minimization in (8), we find that the states which contribute to the partition function have a free-energy density $f^{*}(m, T)$ given either by

$$
\frac{\partial S_{c}(f, T)}{\partial f}\left(f^{*}, T\right)=\frac{m}{T}
$$

if $m$ is small enough (so that the slope at the origin, $\alpha(T)$, be larger than $m / T$ ), or otherwise they are just the low lying states with $f^{*}=f_{\min }$ (see Fig. 1 ). Therefore, varying $m$ at a fixed temperature $T<T_{c}$, we have a phase transition at a critical value of $m=m_{c}(T)$ which is smaller than one.

The general shape of the free energy $\phi(m, T)$ is the one shown in Fig. 2. For $m>m_{c}(T)$ the free energy is constant $\phi(m, T)=f_{\min }(T)$. For $m<m_{c}(T)$ this function allows to compute the configurational entropy through the implicit equation

$$
\begin{gathered}
\frac{\partial[m \phi(m, T)]}{\partial m}=f, \\
\frac{m^{2}}{T} \frac{\partial \phi(m, T)}{\partial m}=S_{c} .
\end{gathered}
$$

Increasing $m$, the transition point $m_{c}$ is determined by the vanishing of the slope $\partial \phi(m, T) / \partial m$.

The following two remarks, which are trivial consequences of the simple nature of the phase transition in these discontinuous systems, are crucial:

- For $m<m_{c}(T)$, the system is in its high-temperature phase, even if $T<T_{c}$. The free-energy $\phi(m, T)$ is that of the high-temperature phase of a system of $m$ constrained copies, a liquid made of molecules built up from $m$ atoms. It can thus be 


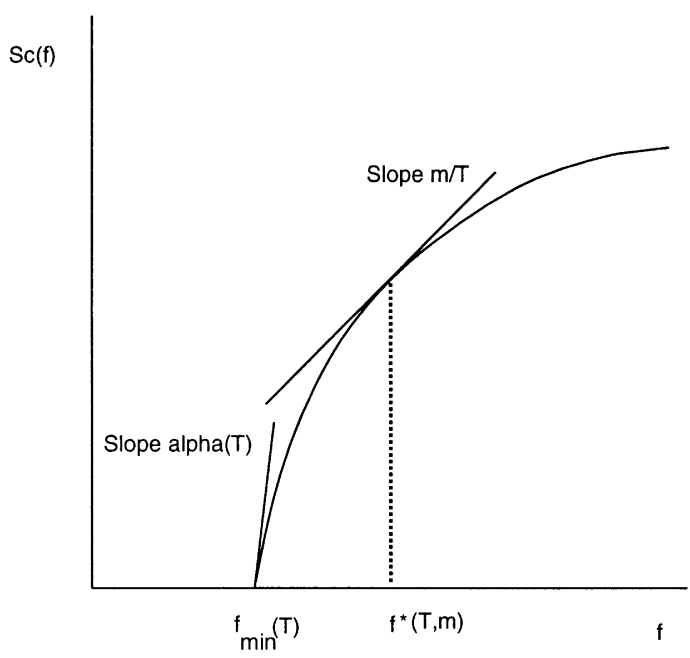

Fig. 1. Qualitative shape of the configurational entropy versus free energy. The whole curve depends on the temperature. The saddle point which dominates the partition function, for $m$ correlated clones, is the point $f^{*}$ such that the slope of the curve equals $m / T$ (for the usual, uncloned, system, $m=1$ ). If the temperature is small enough the saddle point sticks to the minimum $f=f_{\min }$ and the system is in its glass phase.

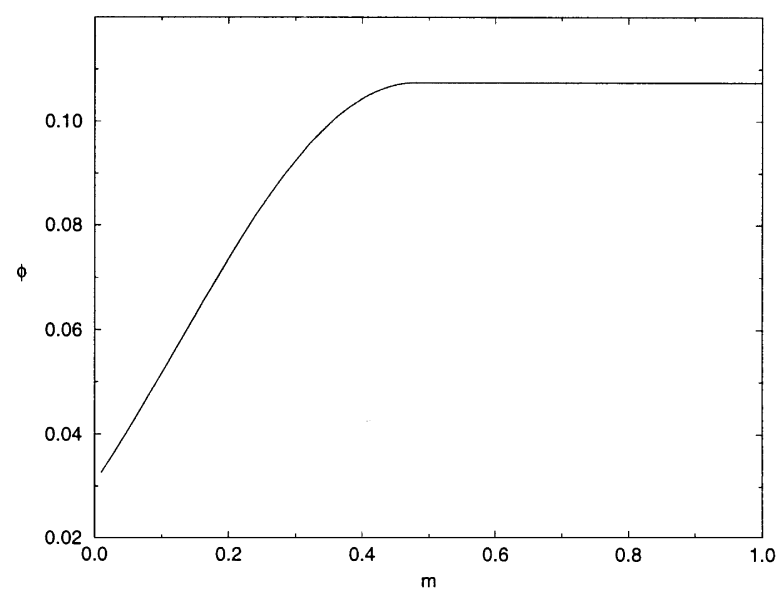

Fig. 2. General shape of the free energy per particle $\phi(m, T)$ of the cloned system, versus the number of clones $m$, at temperatures below the glass transition. The critical value $m=m_{c}(T)$ is identified as the first point where the slope $\partial \phi / \partial m$ vanishes when one increases $m$.

evaluated by any of the methods giving a good description of the high-temperature phase, including series expansion, simulations etc.

- The transition at $m=m_{c}(T)$ is such that the free energy is constant for $m>m_{c}(T)$ (this is a consequence of the transition being due to the sticking of the saddle point in $f$ at the lowest value $f_{\min }$ ). Therefore, the free energy in the glass phase at a temperature $T<T_{c}$ is given by $F(T)=\phi(m=1, T)=\phi\left(m_{c}(T), T\right)$. But the free 
energy is continuous through the transition line, and therefore $\phi\left(m_{c}(T), T\right)$ is the free energy of the high-temperature, 'liquid', phase.

This is the method which has been used in [1] to compute the thermodynamics of the glass. It involves dealing with a cloned system made up of $m$ identical clones constrained to be in the same state, and to do this for any $m$, in such a way as to be able to analytically continue the results to $m<1$. This continuation bears some similarities to, but is different from, the one used in the replica method in order to compute quenched averages. As we shall also need to use these more standard replicas, we shall call the $m$ copies introduced here and in [34,1] some 'clones' of the original problem (we keep the word copies for the case when $m$ is an integer larger or equal to one). One can introduce the clones even for systems which have no quenched in disorder, like structural glasses. The reader will have noticed that the above discussion is a general one which does not rely on the details of the $p$-spin mean-field models; in fact, the procedure of computing the free energy of the cloned and constrained system, $\phi(m)$, in the liquid phase, can be used also in some non-mean-field systems [1], in which it is more difficult to come up with a definition of the metastable states, since these involve fixing some state-dependent boundary conditions in a self-consistent way. The replicas introduced in disordered systems, which are used to handle the average over quenched disorder, can be introduced on top of the clones if needed: in the case of systems with quenched disorder, clones can be replicated! As we shall see, the computation with the fixed number $m$ of coupled clones in the high-temperature phase is equivalent for disordered systems to a 'one step rsb' computation. This has been first found in some computations of neural networks [38] and is expected on general grounds [34].

We would like to also comment on the alternative method which has been proposed independently for the study of the glass phase [35]. It also introduces some clones of the system, but it uses one reference system which is supposed to be thermalized in the glass phase, and then studies the free energy of some other systems, constrained to be at a fixed distance of the reference one. This is a powerful method which has the advantage of allowing to study of metastable states in the high-temperature phase, as has been demonstrated in [36,37]. It has the drawback of requiring to have one system thermalized in the glass phase, which is not easy to achieve. The strategy of cloning discussed here, which has been used in [34,1] has the advantage of involving only computations in the liquid phase, but its scope is probably limited to the study of equilibrium thermodynamics.

\subsection{Two coupled copies}

We have seen in the previous section how a set of $m$ clones constrained to stay in the same state can allow for the computation of thermodynamical properties of glasses. The question we address now is how to achieve this constraint. In order to try and constrain the $m$ clones to be in the same state, we shall introduce an attractive coupling between the various clones. For spin systems, calling $s_{i}^{a}$ the spin at site $i$ in clone $a$, 
this coupling could be for instance:

$$
-\varepsilon \sum_{1 \leqslant a<b \leqslant m} \sum_{i=1}^{N} s_{i}^{a} s_{i}^{b} .
$$

The detailed structure of this coupling is to some extent irrelevant, but it should satisfy the following constraints: it is extensive, in that it contributes to the energy at order $N$, and it is proportional to $\varepsilon$. What we shall be mostly interested in is taking the double limit $\lim _{\varepsilon \rightarrow 0} \lim _{N \rightarrow \infty}$. But it will also turn out to be useful to study the phase diagram of the coupled system for general $\varepsilon$. This we shall do in the next section for the $p$-spin models, but let us first try to get a qualitative understanding of the phase diagram in general, based only on the existence of the configurational entropy. The various parameters in our problem are the number of clones $m$, the temperature $T$, and the coupling between clones $\varepsilon$.

Let us first consider the case of two coupled copies, $m=2$, in the limit of small $\varepsilon$. There can be several contributions to the partition function:

- When the two systems fall into the same state, we have

$$
Z_{=} \simeq \int_{f_{\min }(T)} \mathrm{d} f \mathrm{e}^{N\left[S_{c}(f, T)-2 f / T+\varepsilon q_{1} / T\right]} \equiv \mathrm{e}^{-2 N \phi_{=} / T},
$$

where $q_{1}$ is the typical self overlap of a state.

- When they are in different states, we have

$$
Z_{\neq} \simeq \int_{f_{\min }(T)} \mathrm{d} f \mathrm{e}^{N\left[2 S_{c}(f, T)-2 f / T\right]} \equiv \mathrm{e}^{-2 N \phi_{7} / T} .
$$

There exist several temperature regimes:

- If $T<T_{c}(0)$, both contributions are dominated by the boundary $f=f_{\min }$, giving $\phi_{=}=f_{\min }-\varepsilon q_{1} / 2$ and $\phi_{\neq}=f_{\min }$. Therefore, for any positive $\varepsilon$, we have $\phi_{=}<\phi_{\neq}$ and the leading contribution is due to systems falling into the same state. The system is in a correlated phase because the overlap between the two copies is non-zero, and it is in the glass phase because the free energy sticks at $f_{\min }$.

- When $T_{c}(0)<T<T_{2}$, where $T_{2}$ is the temperature at which $\alpha\left(T_{2}\right)=2 / T_{2}$, we have $\alpha(T)<2 / T$. Thus the contribution $Z_{=}$is dominated by the boundary and $\phi_{=}=$ $f_{\text {min }}-\varepsilon q_{1} / 2$. The contribution $Z_{\neq}$is dominated by a non-trivial saddle point $f^{*}$ such that $\left(\partial S_{c} / \partial f\right)\left(f^{*}\right)=1 / T$. The corresponding free energy is $\phi_{\neq}=f^{*}-T S_{c}\left(f^{*}\right)$. Depending on the value of $\varepsilon$, the minimal free energy will be either $\phi_{=}$, in which case the system is in a correlated glass phase, or $\phi_{\neq}$, in which case the system is in an uncorrelated liquid phase. Because of the concavity of the $S_{c}(f)$ curve, $f^{*}-T S_{c}\left(f^{*}\right)<f_{\min }$ and therefore the 'liquid' saddle point dominates at $\varepsilon$ small enough. The critical value of $\varepsilon$ locating the transition to the glass phase, with the two systems sticking together, is

$$
\varepsilon_{c}=\left(f_{\min }-f^{*}+T S_{c}\left(f^{*}\right)\right) / q_{1} .
$$

This whole discussion holds only at small $\varepsilon$, and in this region one can locate the behavior of the transition curve in the neighborhood of the point $T=T_{c}, \varepsilon=0$, by using 
the following expansion of the configurational entropy at $f>f_{\min }$ :

$$
S_{c}(f)=\alpha(T)\left(f-f_{\min }(T)\right)-\frac{\gamma(T)}{2}\left(f-f_{\min }(T)\right)^{2}+\mathrm{O}\left(\left(f-f_{\min }(T)\right)^{3}\right) .
$$

We have then

$$
f^{*} \simeq f_{\min }+\frac{1}{\gamma(T)}(\alpha(T)-1 / T)
$$

In the neighborhood of the point $T=T_{c}, \varepsilon=0$, the generic behavior is that $\alpha(T)-1 / T$ is linear in $T-T_{c}(0)$. Writing $\alpha(T)-1 / T \sim g\left(T-T_{c}(0)\right)$, we get finally

$$
T_{c}(\varepsilon)-T_{c}(0) \sim \sqrt{\frac{\gamma q_{1}}{T_{c}(0) g^{2}}} \sqrt{\varepsilon}
$$

This discussion suggest that, in the limit of small coupling $(\varepsilon \rightarrow 0)$, the system of two coupled copies will have a transition to the glass state at a temperature which depends continuously on $\varepsilon$, with a square root singularity. This transition is clearly identified by a first-order jump in the correlation between the two systems, defined here as the overlap. Such a system with two copies may thus be a good candidate for the numerical determination of $T_{c}(0)$, as already discussed in [36].

Let us also notice that there might, in principle, exist another phase of the system, with the two copies correlated, but being in the liquid phase. (This is the phase of the molecular liquid.) This can exist whenever $T_{2}<T$ : The contribution $Z_{\neq}$is then dominated by a non-trivial saddle point $f_{\neq}^{*}$ such that $\left(\partial S_{c} / \partial f\right)\left(f_{\neq}^{*}\right)=1 / T$. This is also the case of the contribution $Z_{=}$, with a saddle point $f_{=}^{*}$ such that $\left(\partial S_{c} / \partial f\right)\left(f_{=}^{*}\right)=2 / T$. Concavity implies that $\phi_{\neq}<\phi_{=}$at small enough $\varepsilon$. Therefore, the system is in its uncorrelated liquid phase for $\varepsilon$ small enough, at all temperatures $T>T_{c}$ : in a system with two coupled copies one does not see the correlated liquid phase at small $\varepsilon$. If one could use these small $\varepsilon$ computations to induce some conclusions for finite $\varepsilon$, they would hint at a transition, in the liquid phase, between the uncorrelated one at small $\varepsilon$ and the correlated one at larger $\varepsilon$.

In fact, for very large $\varepsilon$ the two coupled systems will be in the same configuration, giving a partition function $Z=\int \exp (-2 \beta H)$. Therefore, this system will have a usual glass transition at a static temperature

$$
\lim _{\varepsilon \rightarrow \infty} T_{c}(\varepsilon)=2 T_{c}(0)
$$

and this transition will be a discontinuous glass transition, therefore it will have no entropy jump.

The explicit computations in the $p$-spin models will confirm the various aspects of the phase diagram of the two copies system that we have predicted here. We expect that these are general features which will be found in all the glass phases. 


\subsection{Coupled clones}

Let us now study the main aspects of the phase diagram that one can expect when there is a general number, $m$, of clones. As we have seen above, the system can be, for small $\varepsilon$, in any of the following three possible phases:

- A correlated glass phase in which the free energy is $\phi=f_{\min }-(m-1) \varepsilon q_{1} / 2$.

- A correlated liquid phase in which the free energy is the saddle point of $\phi=f-$ $(T / m) S_{c}(f, T)-(m-1) \varepsilon q_{1} / 2$, and the extremum value $f^{*}$ is greater than $f_{\min }$.

- An uncorrelated liquid phase in which the free energy is the saddle point of $\phi=$ $f-T S_{c}(f, T)$ and the extremum value $f^{*}$ is greater than $f_{\min }$.

One has to distinguish two regions in $m$ space.

If $m>1$, the discussion is very similar to the one we did above for the $m=2$ phase, and we expect that at small $\varepsilon$ the system will have a transition from an uncorrelated liquid phase at $T>T_{c}$ to a correlated glass phase at $T<T_{c}$.

If $m<1$ the situation is different, basically because the interesting part of the phase diagram now lies in the $T<T_{c}$ region. When $T<T_{c}$, the uncorrelated liquid cannot exist because $\alpha(T)>1 / T$. Therefore, one expects a transition from the correlated liquid to the correlated glass, at a temperature such that $\alpha(T)=m / T$. This is precisely the transition which was discussed in the previous section. So our conclusion is that the $\varepsilon$ coupling, for small $\varepsilon$ and $T<T_{c}$, is able to polarize the system in the same state provided $m<1$, and thus it realizes the constrained system that was discussed in Section 2.3 and allows for the computation of the glass thermodynamics from liquid computations.

\section{Explicit computations with the $p$-spin model}

We now move on to some explicit computations of the phase diagram of the cloned p-spin model.

\subsection{The usual replica solution}

We work with a spherical spin glass model [23] involving $N$ spins $S_{i}$ with the spherical constraint $\sum_{i} S_{i}^{2}=N$ and an energy given by

$$
H=-\sum_{i_{1}<\ldots<i_{p}} J_{i_{1} \ldots i_{p}} S_{i_{1}} \ldots S_{i_{p}},
$$

where the random couplings $J_{i_{1} \ldots i_{p}}$ are independent Gaussian random variables with zero mean and variance $p ! /\left(2 N^{p-1}\right)$. In the explicit computations below, and for all the numerical results, we shall use the value $p=4$; however, all the systems with $p>2$ have the same qualitative phase diagram. We shall not reproduce here the various techniques which have already been used to study this system, but just state some results of interest for our discussion, referring the reader to the original literature $[23,24,39,40]$. 


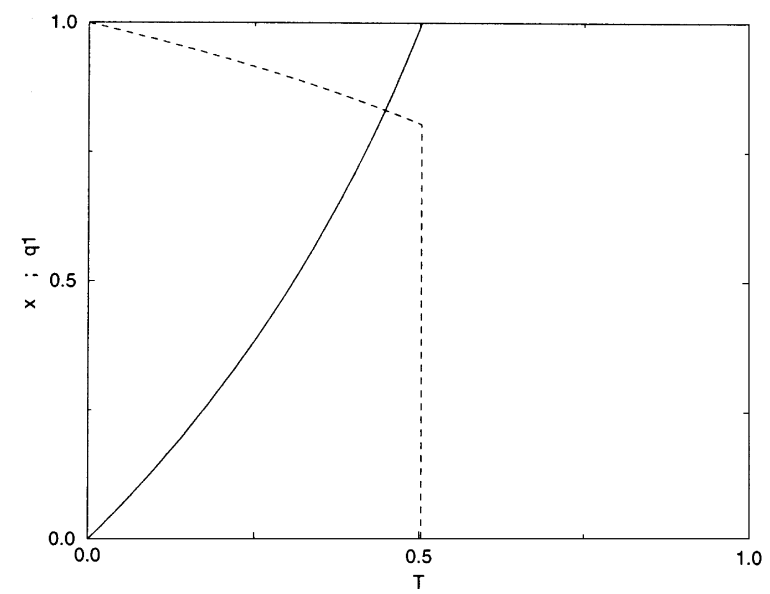

Fig. 3. Replica solution of the $p=4$ model at equilibrium. We plot the Edward-Anderson order parameter $q_{1}$ (dashed line) and the one step rsb parameter $x$ (full line) versus the temperature. The transition is at the temperature $T=0.503$.

The replica solution shows the existence of a transition from a replica symmetric phase at $T>T_{c}$ to a phase with one step rsb. The breaking involves a parametrization of the overlap matrix between $n$ replicas, $Q_{a b}=(1 / N) \sum_{i}\left\langle S_{i}^{a} S_{i}^{b}\right\rangle$, which has a one-step rsb Parisi structure [30] with a diagonal element equal to one, the elements inside diagonal blocks of size $x$ equal to $q_{1}$, and all the other elements equal to zero. The free energy is

$$
F=-\frac{\beta}{4}\left[1-(1-x) q_{1}^{p}\right]+\frac{1-x}{2 \beta x} \log \left(1-q_{1}\right)-\frac{1}{2 \beta x} \log \left[1-(1-x) q_{1}\right]
$$

and should be maximised over $q_{1}$ and over $x$ in the interval $[0,1]$.

The high-temperature phase is obtained when $x \rightarrow 1$. In the high-temperature phase $q_{1}=0$ and the free energy is just $-\beta / 2$.

The parameter $q_{1}$ is interpreted as the Edwards-Anderson (EA)-order parameter, characteristic of the degree of spontaneous ordering inside each state: $q_{1}=(1 / N) \sum_{i}\left\langle S_{i}\right\rangle_{\alpha}^{2}$. The low-temperature phase has an EA-order parameter $q_{1} \neq 0$, and $q_{1}$ jumps discontinuously at $T_{c}$, although the transition is thermodynamically of second order, with a continuous entropy and energy (this is possible because $x \rightarrow 1$ at the transition [6]). The parameter $x$ is characteristic of the distribution of free energies of the low lying states $[21,30]$.

The result for $p=4$ is summarized in Fig. 3, which plots the value of the breakpoint parameter $x$ and the EA parameter versus temperature. The transition temperature, identified from $x=1$, is $T_{c}=0.503$. The solution with $x>1$ is interesting for the study of metastable states and barriers [24], but here we stay within the framework of the equilibrium measure where it does not play any role. 


\subsection{Two coupled copies: exact solution and phase diagram}

We now turn to a coupled system with $m=2$ identical copies of the original problem, two sets of spins $S_{i}$ and $S_{i}^{\prime}$, characterized by the energy

$$
H=-\sum_{i_{1}<\ldots<i_{p}} J_{i_{1} \ldots i_{p}}\left(S_{i_{1}} \ldots S_{i_{p}}^{\prime}+S_{i_{1}}^{\prime} \ldots S_{i_{p}}^{\prime}\right)-\varepsilon \sum_{i} S_{i} S_{i}^{\prime} .
$$

The thermodynamics of a cloned $p$-spin model with some coupling between the clones has already been studied in [24] in order to understand the structure of metastable states and barriers. We shall borrow from this paper the form of the free energy of the coupled system, computed with the replica method (replicated clones), within the one step rsb formalism. The replica formalism introduces $n$ replicas of each of the two spin systems. The free energy is expressed in terms of a self-overlap matrix between $n$ different replicas of the spins $S_{i}, Q_{a b}=(1 / N) \sum_{i}\left\langle S_{i}^{a} S_{i}^{b}\right\rangle=(1 / N) \sum_{i}\left\langle S_{i}^{\prime a} S_{i}^{\prime b}\right\rangle$. It also involves the correlations between the replicas of the two sets of spins, $P_{a b}=(1 / N) \sum_{i}\left\langle S_{i}^{a} S_{i}^{\prime b}\right\rangle$. Within the one step rsb solution, which was shown to be correct in [24], the matrix $Q$ is parametrized exactly as in the previous case by the value of its elements, $q_{1}$, inside the diagonal blocks of size $m$, while the matrix $P$ is parametrized by the value of its elements, $p_{1}$, inside the diagonal blocks of size $m$, and the value $\tilde{p}$ of its diagonal elements (all other elements being 0 ). In terms of these order parameters, the free energy is given by

$$
\begin{aligned}
F= & -\beta\left[f(1)+f(\tilde{p})-(1-x)\left(f\left(q_{1}\right)+f\left(p_{1}\right)\right)\right] \\
& -\frac{1}{2 \beta} \frac{1}{x}\left[\log \left(1-\tilde{p}+(1-x)\left(p_{1}-q_{1}\right)\right)+\log \left(1+\tilde{p}-(1-x)\left(p_{1}+q_{1}\right)\right)\right] \\
& +\frac{1}{2 \beta} \frac{1-x}{x}\left[\log \left(1-\tilde{p}+p_{1}-q_{1}\right)+\log \left(1+\tilde{p}-\left(p_{1}+q_{1}\right)\right)\right]-\varepsilon \tilde{p},
\end{aligned}
$$

where $f(r) \equiv r^{p} / 2$. We have two real copies, so the free energy must be minimized with respect to $\tilde{p}$. But we have $n \rightarrow 0$ replicas to handle the quenched average, so the free energy must be maximized with respect to $p_{1}, q_{1}, x[30]$.

The interpretation of the various order parameters is as follows. The system may freeze into a correlated glass phase. This will be signalled by the Edwards-Anderson-like order parameters

$$
q_{1}=\frac{1}{N} \sum_{i}\left\langle S_{i}\right\rangle_{\alpha}\left\langle S_{i}^{\prime}\right\rangle_{\alpha}, p_{1}=\frac{1}{N} \sum_{i}\left\langle S_{i}\right\rangle_{\alpha}\left\langle S_{i}\right\rangle_{\alpha}
$$

On the other hand, for any finite $\varepsilon$, and both in the high- and low-temperature phase, the degree of correlations between the two systems is measured by

$$
\tilde{p}=\frac{1}{N} \sum_{i}\left\langle S_{i} S_{i}^{\prime}\right\rangle
$$




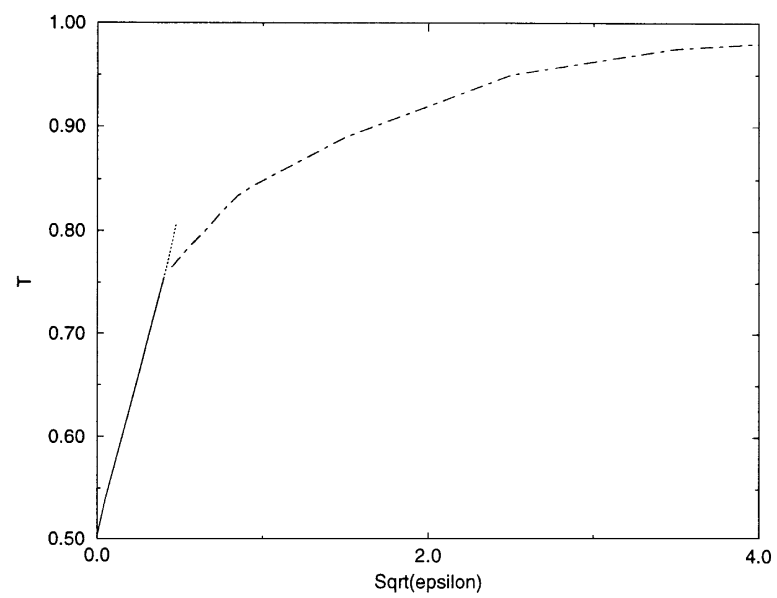

Fig. 4. Phase diagram in the plane $\sqrt{\varepsilon}, T$, for a system of two coupled copies of a $p$-spin model with coupling $\varepsilon$, and $p=4$. Full and dashed-dotted lines: the glass transition temperature $T_{c}$, identified by a jump in the Edwards-Anderson parameter, which is zero in the high-temperature phase, and jumps discontinuously to a positive value in the low-temperature phase. The full line is a first-order transition with a latent heat and a jump in the correlation (see text), while the dashed-dotted line is a 'discontinuous spin glass' transition with no latent heat, no jump in the correlation, but a jump in the EA-order parameters. The dotted line is a first-order transition line, ending at a second-order critical point, separating two regions of the liquid with different correlations between the two copies.

The extremisation of the free energy at $p=4$ and finite $\varepsilon$, performed by a simple grid search followed by a minimization procedure, gives the phase diagram plotted in Fig. 4.

Above the full and dashed-dotted lines, the system is in a liquid phase, where the EA-order parameters are $q_{1}=p_{1}=0$. The correlation $\tilde{p}$ is non-zero, but it goes to zero in the limit of $\varepsilon \rightarrow 0$. Below the full and the dashed-dotted lines the system is in the glass phase, with $q_{1}=p_{1}>0$. The correlation $\tilde{p}$ is non-zero, and it remains finite when $\varepsilon \rightarrow 0$. Therefore, the $\varepsilon \rightarrow 0$ transition is between an uncorrelated liquid phase at high temperature and a correlated glass phase at low temperatures. It occurs at the temperature $T_{c}(0)=0.503$, with some small $\varepsilon$ corrections which are of order $\sqrt{\varepsilon}$, as anticipated in our general discussion of Section 2.3. This glass transition is a first-order transition on the portion with small enough $\varepsilon$ (full line). On this portion the various order parameters $q_{1}, p_{1}, \tilde{p}, x$ jump discontinuously at the transition. In particular, the correlation parameter $\tilde{p}$ is discontinuous on this transition, which should make it relatively easy to detect numerically (letting aside metastability effects), and there is also a latent heat. The glass transition at larger $\varepsilon$ (dashed-dotted line) is of the second-order type from the thermodynamic point of view. It has a jump in the EAorder parameter $q_{1}=p_{1}$, but the correlation parameter $\tilde{p}$ and the entropy are continuous at the transition; it is thus a transition which is exactly of the 'discontinuous spin glass type', as it is known to occur both for the single system $\varepsilon=0$, as well as for the infinitely coupled system $\varepsilon \rightarrow \infty$.

The first-order transition line goes on into the liquid phase, where there is a portion (dotted line) which is a first-order transition line. This transition has nothing to do with 


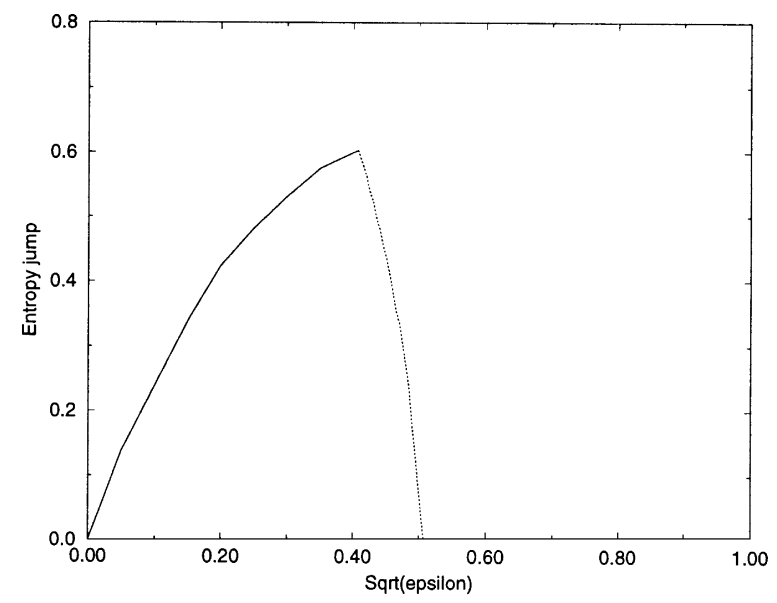

Fig. 5. Discontinuity of the entropy along the two lines of first-order transition (full line and dotted line) in Fig. 4. The transition becomes of second order (continuous entropy) at the two end points: the end point at $\varepsilon=0$ which is the usual glass transition, and the critical point for the decorrelation transition in the liquid phase. The entropy jump is continuous along the triple point, but its derivative is discontinuous.

the glass transition: on both sides of the line the EA-order parameter is $q_{1}=p_{1}=0$. It is a transition in the liquid phase between a regime of strong correlations between the two copies (large $\tilde{p}$ ) when $\varepsilon$ is larger than the transition value, and a regime of smaller correlations, we shall call it the correlation transition. Along this line the entropy is discontinuous. However, these two transition lines (full line and dotted line) are not the analytic continuation one from the other: this is clearly seen from Fig. 5 which plots the jump of entropy along the two discontinuous transition lines. The latent heat is continuous across the triple point when one follows the discontinuous transition lines, but its derivative is discontinuous across the triple point. The first-order correlation transition ends up in the liquid phase at a critical point where the correlation transition becomes of second order. This correlation transition is very similar to the familiar liquid-gas transition.

It is interesting to notice how the glass transition is transmuted by going from a pure system to a system with two copies. In the pure system it is a 'discontinuous spin glass' transition which is difficult to identify, being only signalled by a jump of the EA-order parameter. When we switch on a small coupling $\varepsilon$ between the copies, we get a real first-order transition with an easily measurable jump in $\tilde{p}$ and a specific heat. This was already noticed by Franz and Parisi in their study of this same problem, as well as in their study of two constrained systems, one being thermalized in the glass phase [36]. One outcome of the present computation is that the glass transition is present at all $\varepsilon$ : there is no way one can go continuously from the high-temperature phase to the glass phase without crossing a phase transition. This transition can be either of the 'discontinuous spin glass' nature when the coupling $\varepsilon$ is either zero or large, or it may be first order when the coupling is small enough and non-zero, because it is then accompanied by a correlation transition. 


\subsection{Studying the glass through the cloned liquid}

We now move to the case in which we have $m$ coupled clones, and study specifically the case $m<1$. The general solution could be studied from the formulas derived in [24], but here we want to concentrate on the test of the general method described above, which states that the thermodynamics of the glass can be studied from a high-temperature computation of a correlated system. We thus consider the $m$ systems with a fixed correlation between any of the $m(m-1) / 2$ pairs:

$$
\frac{1}{N} \sum_{i} S_{i}^{a} S_{i}^{b}=q
$$

and compute the partition function

$$
\begin{aligned}
Z= & \int \prod_{a=1}^{m}\left(\prod_{i} \mathrm{~d} S_{i}^{a} \delta\left(\sum_{i}\left(S_{i}^{a}\right)^{2}-N\right)\right) \prod_{a<b} \delta\left(\frac{1}{N} \sum_{i} S_{i}^{a} S_{i}^{b}-q\right) \\
& \times \exp \left(-\sum_{i_{1}<\ldots<i_{p}} J_{i_{1} \ldots i_{p}} \sum_{a=1}^{m} S_{i_{1}}^{a} \ldots S_{i_{p}}^{a}\right)
\end{aligned}
$$

As we perform only a high temperature computation, we do not need to introduce the replicas in order to perform the quenched average. The simplest method is to notice that the annealed average is exact in the high-temperature phase (or we can also expand $Z$ in a high temperature series). We find a free-energy $\phi=-\log Z /(\beta N m)$ equal to:

$$
\phi=-\frac{\beta}{4}\left[1-(1-m) q^{p}\right]+\frac{1-m}{2 \beta m} \log (1-q)-\frac{1}{2 \beta m} \log [1-(1-m) q] .
$$

This is exactly the free energy of a single, uncloned system, when it is studied through the introduction of replicas in (21). Our number of clones $m$ is equivalent to the breakpoint $x$ in the one step rsb Ansatz, and the correlation $q$ is equivalent to the matrix element $q_{1}$ in the replica method. As explained in the previous section, this could be expected on general grounds [34]. It also implies that the proposed method of the high-temperature expansion of a cloned system works. In fact if one studies, for $m<1$, the free energy $\phi$ using the general method outlined in Section 2.3, one gets the phase diagram in the plane $m, T$ shown in Fig. 6 . The value of the critical temperature $T_{c}(m)$ is the inverse function of the $x(T)$ curved obtained with the replica solution, and the thermodynamics deduced from Section 2.3 is automatically correct.

The interesting point of this study is the structure of the phase diagram of Fig. 6. It confirms our expectations from the above general discussion: the transition is rather different depending on whether $m$ is larger or smaller than one. When $m<1$ the glass transition is between a correlated liquid and a glass. Therefore, the high-temperature phase that must be studied in order to compute the properties of the glass is that of 


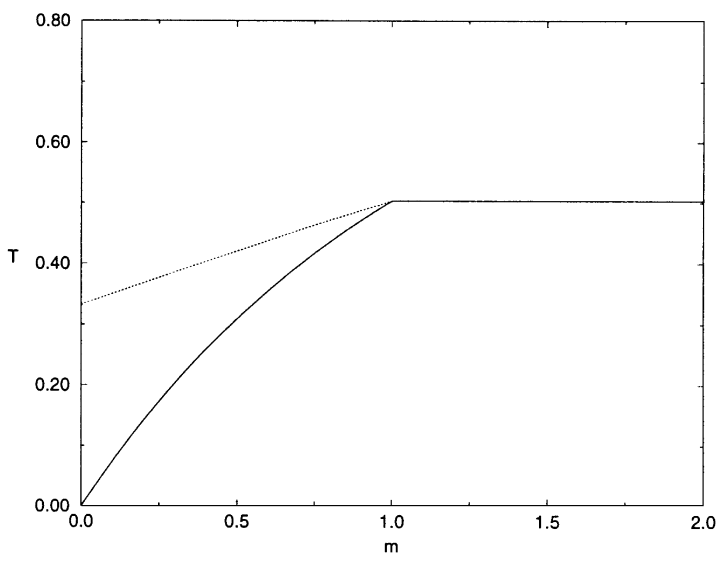

Fig. 6. Phase diagram of the cloned $p$-spin glass with $p=4$ at small $\varepsilon$ in the plane $m$ (number of clones) temperature. The diagram is obtained from maximising (if $m<1$ ) or minimizing the free energy of $m$ clones, with the high-temperature-liquid-free energy involving only the correlation $q$ between the clones. Above the full line the system is in the liquid phase, below it is a glass. The free energy along the transition line at $m<1$ can be obtained from a high-temperature computation in a correlated liquid. As the free energy in the glass phase is independent of $m$, this allows to compute the free energy in the glass phase. The dotted line is a first order transition line between a liquid which is coupled, with a finite value of $q$, below the dotted line, and one where the clones are uncoupled, $(q)$, above the line.

the correlated liquid. ${ }^{3}$ When $m>1$ the transition is between an uncorrelated liquid and a glass. This explains some of the previous failures and successes of recent works on glasses using this clonation idea. In [41] the transition in soft or hard sphere systems was studied as one from an uncorrelated liquid to a glass. This allowed to get a good result for the transition temperature, but it did not give the correct glass thermodynamics, because this glass thermodynamics should be studied from a study of a correlated liquid, not from an uncorrelated one. The correct procedure was performed in [1] through a study of a molecular liquid.

\section{Conclusion}

We have tested the general method of [1] on the solvable case of the $p$-spin model. It works well in the sense that the study of a 'molecular liquid' with $m<1$ clones allows to obtain the exact result for the thermodynamics of the glass phase. Obviously, the computations which are performed with the clones in their high-temperature phase are equivalent to the direct 'one step replica symmetry breaking' solution of the problem: the clones are basically equivalent to replicas [34,38]. However, one should not forget that the clones give us a method to compute the thermodynamic properties of glasses

\footnotetext{
${ }^{3}$ Notice that the phase of uncorrelated liquid extends here in the region $T<T_{c}$, contrarily to the general picture of Section 2.3. The reason is that we draw here the phase diagram as obtained from the high temperature computation which is unable to detect a glass transition at $T_{c}$.
} 
even in systems without any quenched-in disorder, where there is no way to introduce the replicas. In fact, we believe that the method of replica symmetry breaking, although it was historically introduced in order to compute some quenched averages, involving the logarithm of a partition function, is much more general. At the one step level it is able to address any kind of problem (with or without disorder) where the low lying minima are uncorrelated, with a distribution which is unbounded but decreases faster than any power law [22]. This is very interesting since these problems build up one natural universality class for extreme events statistics. The question of whether a given problem (e.g. a given type of structural glass) falls into this universality class is a difficult one. If it does not, it means that there will be another phase transition which forbids the direct study of the glass from that of the cloned liquid (a system with two step breaking could be studied by cloning the clones... ). This is very probably what happens in the Edwards Anderson model [42]. The case of structural glasses, if it is confirmed that it belongs to this universality class of systems which are solved by cloning, (the favorable indications are reviewed in [1]), is thus a much simpler one. Yet it would also be interesting to try to carry out a similar method on finite-dimensional spin glasses, in order to see the predictions, e.g. for the ground state energy.

\section{Acknowledgements}

It is a pleasure to thank G.Parisi for many discussions and comments. This research was supported in part by the National Science Foundation under Grant No. PHY94-07194.

\section{References}

[1] M. Mézard, G. Parisi, cond-mat/9807420 and in preparation.

[2] A.W. Kauzman, Chem. Rev 43 (1948) 219.

[3] G. Adams, J.H. Gibbs, J. Chem. Phys 43 (1965) 139.

[4] J.H. Gibbs, E.A. Di Marzio, J. Chem. Phys. 28 (1958) 373.

[5] B. Derrida, Phys. Rev. B 24 (1981) 2613.

[6] D.J. Gross, M. Mézard, Nucl. Phys. B 240 (1984) 431.

[7] T.R. Kirkpatrick, P.G. Wolynes, Phys. Rev. A 34 (1986) 1045.

[8] T.R. Kirkpatrick, D. Thirumalai, Phys. Rev. Lett. 58 (1987) 2091.

[9] T.R. Kirkpatrick, D. Thirumalai, Phys. Rev. B 36 (1987) 5388.

[10] T.R. Kirkpatrick, D. Thirumalai, P.G. Wolynes, Phys. Rev. A 40 (1989) 1045.

[11] C.A. Angell, Science 267 (1995) 1924.

[12] P. De Benedetti, Metastable Liquids, Princeton University Press, Princeton, 1997.

[13] J. Jäckle, Rep. Prog. Phys. 49 (1986) 171.

[14] G. Parisi, Proc. ACS Meeting, Orlando, 1996, cond-mat/9701068, Lecture given at the Sitges Conf., June 1996 cond-mat/9701034 and Lectures given at the Varenna summer school 1996, cond-mat/9705312.

[15] G. Parisi, cond-mat/9712079.

[16] G. Parisi, Phys. Rev. Lett. 50 (1983) 1946.

[17] G. Toulouse, in: Heidelberg Colloquium on Spin Glasses, I. Morgenstern, L. van Hemmen (Eds.), Lecture Notes in Physics, vol. 192, Springer, Berlin, 1983.

[18] S. Caracciolo, G. Parisi, S. Patarnello, N. Sourlas, Europhys. Lett. 11 (1990) 783. 
[19] S. Franz, G. Parisi, M.A. Virasoro, J. Phys. I (France) 2 (1992) 1969.

[20] M. Mézard, J. Physique 51 (1990) 1831.

[21] M. Mézard, G. Parisi, M.A. Virasoro, J. Physique Lett. 46 (1985) L21.

[22] J.-P. Bouchaud, M. Mézard, J. Phys. A 30 (1997) 7337.

[23] A. Crisanti, H.-J. Sommers, Z. Phys. B 87 (1992) 341.

[24] J. Kurchan, G. Parisi, M.A. Virasoro, J. Phys. I France 3 (1993) 1819.

[25] J.-P. Bouchaud, M. Mézard, J. Physique I (France) 4 (1994) 1109.

[26] E. Marinari, G. Parisi, F. Ritort, J. Phys. A 27 (1994) 7615.

[27] E. Marinari, G. Parisi, F. Ritort, J. Phys. A 27 (1994) 7647.

[28] P. Chandra, L.B. Ioffe, D. Sherrington, Phys. Rev. Lett. 75 (1995) 713.

[29] P. Chandra, M.V. Feigelman, L.B. Ioffe, Phys. Rev. Lett. 76 (1996) 4805.

[30] M. Mézard, G. Parisi, M.A. Virasoro, Spin Glass Theory and Beyond, World Scientific, Singapore, 1987.

[31] R. Palmer, in: Heidelberg Colloquium on Spin Glasses, I. Morgenstern, L. van Hemmen (Eds.), Lecture Notes in Physics, vol. 192, Springer, Berlin, 1983.

[32] M. Mézard, G. Parisi, N. Sourlas, G. Toulouse, M. Virasoro, Phys. Rev. Lett. 52 (1984) 1156.

[33] M. Mézard, G. Parisi, N. Sourlas, G. Toulouse, M. Virasoro, J. Physique 45 (1984) 843.

[34] R. Monasson, Phys. Rev. Lett. 75 (1995) 2847.

[35] S. Franz, G. Parisi, J. Physique I 5 (1995) 1401.

[36] S. Franz, G. Parisi, Phys. Rev. Lett. 79 (1997) 2486, and cond-mat/9711215.

[37] M. Cardenas, S. Franz, G. Parisi, cond-mat/9712099.

[38] R. Monasson, D. O'Kane, Europhys. Lett. 27 (1994) 85.

[39] A. Crisanti, H.-J. Sommers, J. Phys. I (France) 5 (1995) 805.

[40] A. Crisanti, H. Horner, H.-J. Sommers, Z. Phys. B 92 (1993) 257.

[41] M. Mézard, G. Parisi, J. Phys. A 29 (1996) 6515.

[42] E. Marinari, G. Parisi, F. Ricci-Tersenghi, J.J. Ruiz-Lorenzo, Violation of the Fluctuation Dissipation Theorem in Finite Dimensional Spin Glasses, cond-mat /9710120. 